\title{
Transcutaneous electrical acupoint stimulation (TEAS) ameliorates chemotherapy-induced bone marrow suppression in lung cancer patients
}

\author{
Lili Hou', Fen Gu², Guanghui Gao ${ }^{3}$, Caicun Zhou ${ }^{3}$ \\ ${ }^{1}$ Department of Nursing, Shanghai Pulmonary Hospital of Tongji University, Shanghai 200043, China; ${ }^{2}$ Respiratory Department, Shanghai \\ Pulmonary Hospital of Tongji University, Shanghai 200043, China; ${ }^{3}$ Department of Oncology, Shanghai Pulmonary Hospital of Tongji University, \\ Shanghai 200043, China \\ Contributions: (I) Conception and design: C Zhou, L Hou, G Gao; (II) Administrative support: All authors; (III) Provision of study materials or \\ patients: All authors; (IV) Collection and assembly of data: All authors; (V) Data analysis and interpretation: F Gu, G Gao; (VI) Manuscript writing: \\ All authors; (VII) Final approval of manuscript: All authors. \\ Correspondence to: Caicun Zhou. Department of Oncology, Shanghai Pulmonary Hospital of Tongji University, Shanghai 200043, China. \\ Email: zhoucc2302@163.com.
}

\begin{abstract}
Background: To investigate the effect of percutaneous electrical stimulation on chemotherapy-induced bone marrow suppression in patients with lung cancer.

Methods: From December 2014 to August 2015, one hundred ninety-one non-small cell lung cancer patients with chemotherapy naive were randomly divided into control group, medication group, and transcutaneous electrical acupoint stimulation (TEAS) group. Patients with the control group received routine nursing care, the medication group was treated by oral administration of prophylactic agents, and TEAS group received electrical stimulation of acupoints including Dazhui (DU14), Geshu (BL17), Zusanli (ST36), Sanyinjiao (SP6), and Hegu (LI4). The primary end point was the blood routine indexes and secondary end point was the degree of comfort.

Results: The white blood cell in the TEAS group was significantly higher than the control group on day 8 and day $14(\mathrm{P}<0.05)$. The platelet count in the TEAS group was significantly higher than control group on day 5 , day 8 and day $11(\mathrm{P}<0.05)$. The comfort score in the TEAS group was significantly higher than control group on day $8(\mathrm{P}<0.05)$.

Conclusions: Percutaneous electrical stimulation of acupoints could prevent chemotherapy-induced bone marrow suppression in lung cancer patients and ensure a smooth continuation of chemotherapy.
\end{abstract}

Keywords: Acupoint; bone marrow suppression; chemotherapy; electrical stimulation; lung cancer

Submitted Nov 15, 2016. Accepted for publication Feb 12, 2017.

doi: $10.21037 /$ jtd.2017.03.12

View this article at: http://dx.doi.org/10.21037/jtd.2017.03.12

\section{Introduction}

Lung cancer is the malignant neoplasm with the highest incidence and mortality in China (1) as well as worldwide (2). Data from the National Cancer Prevention and Control Office of the Ministry of Health have shown that the incidence of lung cancer has been increasing every year by $26.9 \%$ in China (1). Globally, the incidence of lung cancer in 2010-2012 was 30.09 people per 10 million (2).
Non-small cell lung cancer (NSCLC) accounts for $80 \%-$ $85 \%$ of all lung cancer cases. Chemotherapy is the preferred treatment method for NSCLC, and gemcitabine plus platinum (GP) is the most common drug combination (1). GP significantly reduces the mortality of NSCLC patients (3-5). However, many patients cannot tolerate the adverse reactions of chemotherapy. Among the most common and most severe adverse reactions is bone marrow suppression, with grade 
III-IV neutropenia reaching percentages of about $20 \%(6,7)$. The more serious the bone marrow suppression, the higher the direct medical cost of chemotherapy (8). Bone marrow suppression is defined as a decrease of blood cell activity in the bone marrow, which is mainly manifested by a decrease of white blood cells and platelets, and less so of red blood cells. Within the chemotherapy, carboplatin decreases white blood cell count by $27 \%$ after 10-14 days of initiation of treatment (9). For nedaplatin, which suppresses the bone marrow function by $80 \%$, making it more toxic than cisplatin. The treatment of bone marrow suppression, which is colony-stimulating factor drugs, can quickly mobilize mature granular cells into the peripheral blood. However, it is costly and often lead to systemic joint pain (10).

At present, TCM uses mainly point injection therapy to treat bone marrow suppression. The theory of traditional Chinese medicine (TCM) considers that chemotherapy has the function of "getting rid of evil pathogens" that injure the viscera, bone marrow, blood, Yin and Yang, resulting in spleen, stomach, liver, and kidney function impairment. Chemotherapy is classified into the category of "attack evil" because of its toxic properties (11). Yu et al. showed that Zusanli acupoint injection with dexamethasone combined with oral tablets significantly increases the white blood cell and neutrophil counts (12). Feng et al. (13) showed that Sanyinjiao acupoint injection enhances the effects of cyclophosphamide by inhibiting tumor growth and relieving bone marrow suppression and the adverse effects on the immune system. Acupuncture and acupoint injection at Zusanli, Sanyinjiao, Xuehai, Qihai, and Guan Yuan have also been reported to improve chemotherapy-induced bone marrow suppression (14). However, the above-mentioned interventions are invasive. Patients reportedly experience pain and present poor compliance to the treatment. Therefore, the clinical efficacy of those interventions needs further investigation.

This study aims to investigate the effects of TEAS on bone marrow suppression in chemotherapy patients with lung cancer. According to the TCM channel and collateral theory, transcutaneous electrical acupoint stimulation (TEAS) would introduced to reduce bone marrow suppression at Dazhui (DU14), Geshu (BL17), Zusanli (ST36), Sanyinjiao (SP6), and Hegu (LI4), which play a role in enriching blood and treating leucopenia (15). Although the literature reports no incompatibility between these acupoints, their combined effects have not been studied yet.

\section{Methods}

\section{Study design}

The research protocol for prospective analysis was approved on October 14th, 2014 by the ethics committee of the Shanghai Pulmonary Hospital, China (Ref. k14-155). This study was a single-center, prospective, randomized trial lasting 28 days. The study protocol was approved by the Chinese Medicine Research of Shanghai Municipal Health and Family Planning Commission (Ref. 2014LP036A). The study was registered from the U.S. Clinical Trial Registry. The registration number of the clinical trial is NCT02663492. A research assistant collected the outcome surveys at baseline before the start of chemotherapy and at day $5,8,11,14,21$, and 28 of the program. The date range for patient recruitment was from December 1st, 2014 to July 31 st, 2015. To recruit the study patients, the investigators explained the purpose of the research. The date range for follow up was 28 days after the first day of chemotherapy. Written informed consent was obtained from all subjects before randomization.

\section{Randomization}

Patients were assigned to the experimental groups using a table of computer-generated random numbers. According to the order of admission, the patients were randomly allocated to each of the three groups: the control group, the medication group, and the TEAS group.

\section{Study protocol}

All the patients were receiving cancer routine care at the time of hospitalization, in terms of disease observation, dietary care, and psychological care. In the event of bone marrow suppression based on the Bone marrow suppression WHO classification, the patient will withdraw from this study.

The control group received routine nursing care while taking into consideration the following points: (I) the observation of the disease: strict observation of the changes in patient condition; (II) dietary care: during chemotherapy, patients were encouraged to eat high-calories, high-protein, high-vitamin, easy-to-digest foods; (III) psychological care: patients were given psychological support and spiritual comfort, and were encouraged to establish confidence and avoid iatrogenic trauma; (IV) vein embolic protection: 


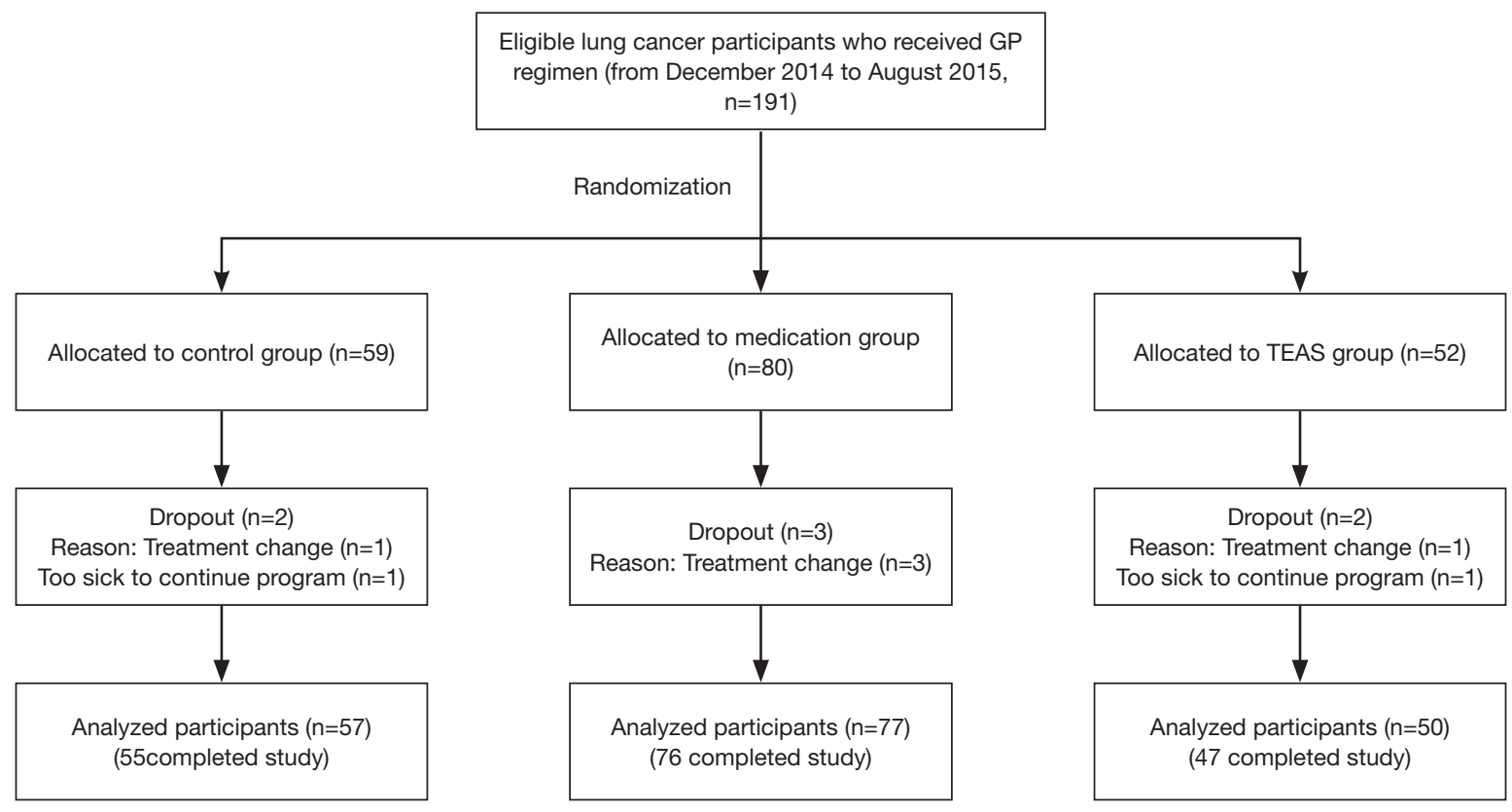

Figure 1 Flow chart of the study.

prevent the occurrence of phlebitis, in strict accordance with aseptic operation principles; and $(\mathrm{V})$ routine use of antiemetics (e.g., ondansetron) and gastroprotective agents (e.g., omeprazole).

The medication group received the following interventions: On the basis of routine nursing care, patients need to take leukemogenic medication such as Diyu Shengbai Pian (a TCM). Nurses should do a good job in medication guidance and health education by educating the patients to take their medicines at the correct dosage and time. In addition, nurses should: follow-up on the registration form, take notes of the follow-up date and times and any complaints or suggestions, give the patients a medication $\log$ and guide them to fill the form (date, time, dose, and patient signature), tell the family members to check the signature and ensure the patient's medication compliance, perform regular blood examinations, and report any adverse reactions to the clinicians in timely fashion.

The TEAS group received preoperative TEAS for $30 \mathrm{~min}$ before the induction of anesthesia in the holding area. After training the intensity, frequency, and complications of nursing to four nurses, they were considered ready to operate the TEAS. On the basis of routine nursing care, acupoint locations were done by TCM clinical experts. TEAS were applied to five pairs of acupoints: Dazhui (DU14), Geshu (BL17), Zusanli (ST36), Sanyinjiao (SP6), and Hegu (LI 4). These acupoints were identified according to the traditional anatomical locations (Figure 1). On days $1,2,3,5,8,11,14,21$, and 28 , TEAS was performed with a dense-disperse frequency of $30 / 100 \mathrm{~Hz}$ and an intensity of 6-15 V for 30 min using the G6805-II (Shanghai Medical Equipment Technology Co. Ltd., Shanghai, China). The optimal intensity was adjusted to maintain a slight twitching of the regional muscle according to individual maximum tolerance. In this research, TEAS was used for each acupoint 30 min per day in a total of 9 days. Each participant should fill in an electrical acupoint stimulation record card, including date, frequency, intensity, and complications. The patients booked any subsequent TEAS appointments to ensure that electrical stimulation of acupoints was performed in accordance with the experimental design of the study. Researchers in the operation process were requested to make timely observations of skin irritation and itching, among others, and to communicate them to patients. There were no adverse reactions in any of the 47 patients of the TEAS group.

\section{Patients}

We enrolled 191 consecutive patients (age range, 34-77 years) from department of oncology in Shanghai pulmonary hospital. The patients were randomized to the control group $(n=59)$, the medication (oral tablets) group $(n=80)$, and the transcutaneous electrical acupoint stimulation (TEAS) 
Table 1 Location of the acupoints used for TEAS

\begin{tabular}{ll}
\hline Acupoint & Location \\
\hline Dazhui (DU14) & Seventh cervical spinous process \\
Geshu (BL17) & Thoracic vertebral spinous process, 1.5 inches away from the midline \\
Zusanli (ST36) & Lateral side of the lower leg, 3 inches under Dubi (S35) and in line with Dubi and Jiexi (ST41) \\
Sanyinjiao (SP6) & Posterior margin of the tibia, close to the edge of the bone \\
Hegu (LI4) & Back of the hand, at the midpoint of the second metacarpal radial side \\
\hline
\end{tabular}

Table 2 Baseline characteristics of the study

\begin{tabular}{|c|c|c|c|c|c|}
\hline Categorical variables & Control $(n=55)$ & Medication ( $n=76$ ) & TEAS $(n=47)$ & $\mathrm{F}$ & $P$ \\
\hline Age (years, mean $\pm S D$ ) & $58.8 \pm 10.72$ & $59.46 \pm 8.14$ & $58.20 \pm 8.30$ & $0.282^{a}$ & 0.755 \\
\hline Course of disease (days) & $10.44 \pm 2.28$ & $10.4 \pm 2.34$ & $10.53 \pm 2.33$ & $1.867^{\mathrm{a}}$ & 0.276 \\
\hline BMI & $26.42 \pm 17.02$ & $22.31 \pm 5.76$ & $24.01 \pm 6.13$ & $1.819^{\mathrm{a}}$ & 0.166 \\
\hline Gender & & & & $1.963^{\mathrm{b}}$ & 0.375 \\
\hline Male & 44 & 61 & 33 & & \\
\hline Female & 11 & 15 & 14 & & \\
\hline Age & & & & $0.137^{b}$ & 0.934 \\
\hline$<60$ years & 25 & 37 & 22 & & \\
\hline$\geq 60$ years & 30 & 39 & 25 & & \\
\hline KPS score & & & & $0.634^{b}$ & 0.728 \\
\hline$\geq 80$ & 54 & 73 & 46 & & \\
\hline$<80$ & 1 & 3 & 1 & & \\
\hline Type & & & & $0.201^{b}$ & 0.995 \\
\hline Adenocarcinoma & 29 & 38 & 24 & & \\
\hline Squamous carcinoma & 17 & 24 & 14 & & \\
\hline Others & 9 & 14 & 9 & & \\
\hline Stage & & & & $0.054^{b}$ & 0.973 \\
\hline I-III & 51 & 70 & 43 & & \\
\hline IV & 4 & 6 & 4 & & \\
\hline
\end{tabular}

${ }^{\mathrm{a}}$, F value; ${ }^{\mathrm{b}}, \chi^{2}$ value. SD, standard deviation; BMI, body mass index.

group ( $\mathrm{n}=52)$. However, 7 patients dropped out of the study because they changed treatment or were too sick to continue the program. Finally, 184 patients including 55 patients of control group, 80 patients of medication group and 50 patients of TEAS group completed the study (Tables 1,2).

The inclusion criteria were as follows: (I) diagnosis of NSCLC, (II) age between 20 and 80 years, (III) chemotherapy naive after diagnosis, (IV) GP regimen as treatment, $(\mathrm{V})$ an expected survival of more than 12 weeks based on a score equal to or greater than 60 points on Karnofsky performance status (KPS) scale, and (VI) the ability to understand and speak Mandarin. The exclusion criteria were as follows: (I) the inability to meet the inclusion criteria, (II) diagnosis of a hematological system tumor, (III) mental illness, (IV) bone marrow suppression prior to chemotherapy, and (V) pregnancy. 


\section{Study outcomes}

The primary end point was the blood routine indexes. The ADVIA120 automatic hematology analyzer (Bayer, Leverkusen of Germany) was used for routine blood examinations of indicators of bone marrow suppression, including red blood cells $\left(\times 10^{12} / \mathrm{L}\right)$, hemoglobin $(\mathrm{g} / \mathrm{L})$, white blood cells $\left(\times 10^{9} / \mathrm{L}\right)$, and platelets $\left(\times 10^{9} / \mathrm{L}\right)$. The tests were performed on the day before the start of chemotherapy and at days $5,8,11,14,21$, and 28 of the study period.

The secondary end point was the degree of comfort. The degree of comfort was assessed on the day before chemotherapy and at days 8 and 28 of the experimental period. The Comfort Scale (questionnaire GCQ, general comfort), which was designed by the American comfort nursing expert, Kolcaba, has a good reliability (Cronbach's alpha $=0.96)(16)$. Content validity index was 0.86 . There are 30 items in this scale, including 5 physical dimensions, 10 mental dimension, 8 social cultural dimensions, and 7 environmental dimensions. Each of the items is classified using a 1-4 Likert scale. The lowest score is 30 points, and the highest score is 120 points. The higher the score, the more comfort the patient is experiencing. A total score of 60 points or less indicates low comfort, a total score of $60-90$ points indicates moderate comfort, and a total score of $>90$ indicates high level of comfort (17). A preliminary experiment was conducted in 30 patients with NSCLC before the first visit, and the reliability coefficient of the scale was calculated; Cronbach's alpha $=0.932$ indicated high internal consistency.

\section{Statistical analyses}

Statistical analysis was performed using SPSS 17.0 (SPSS Inc., Chicago, IL, USA). Enumeration data was presented as absolute quantity. Measurement data are presented as mean \pm SD. One-way analysis of variance was used to compare the patients' age (mean $\pm \mathrm{SD}$ ), course of disease, BMI, white blood cells, platelets, red blood cells, hemoglobin, comfortable score among three groups. Three independent samples nonparametric tests were used to analyze the patients' age ( $<60 v s . \geq 60$ years), KPS score, type and stage. Statistical significance was defined as a $\mathrm{P}$ value $<0.05$.

\section{Results}

\section{Changes in white blood cell counts}

There were no significant differences in the number of white blood cells between groups $(\mathrm{P}>0.05)$ on the day before chemotherapy. The white blood cell count on days 8 and 14 after chemotherapy was significantly higher in the TEAS group than in the control group $(\mathrm{P}<0.05)$. The white blood cell count was significantly higher in the medication group than in the control group $(\mathrm{P}<0.05)$. There were no significant differences in the number of white blood cells between medication and TEAS groups $(\mathrm{P}>0.05)$ (Table 3).

\section{Changes in platelet counts}

There were no significant differences in the number of platelets between groups $(\mathrm{P}>0.05)$ on the day before chemotherapy. On days 5, 8, and 11 , the platelet count was significantly higher in the TEAS group than in the control and medication groups $(\mathrm{P}<0.05)$. On day 21 , the platelet count was significantly higher in the control and TEAS groups than in the medication group $(\mathrm{P}<0.05)$ (Table 4$)$.

\section{Changes in red blood cell counts}

There were no significant differences in the number of red blood cells between groups on any of the studied time points $(\mathrm{P}>0.05)$ (Table 5).

\section{Changes in bemoglobin concentrations}

There were no significant differences in hemoglobin concentration between groups $(\mathrm{P}>0.05)$ on the day before chemotherapy. On day 14 , hemoglobin concentration was significantly higher in the medication groups than in the control group $(\mathrm{P}<0.05)$ (Table 6).

\section{Comfort score}

There were no significant differences in the comfort score between the three groups $(\mathrm{P}>0.05)$ on the day before chemotherapy. On day 8 , the comfort score was significant higher in the TEAS group than in the control group $(\mathrm{P}<0.05)$ (Figure 2).

\section{Discussion}

The findings of this study showed that percutaneous electrical stimulation of acupoints can prevent chemotherapyinduced bone marrow suppression in NSCLC patients. The symptoms of bone marrow suppression include physical decline, fatigue, weakness, sweating, dizziness, tinnitus, palpitation, severe palpitation, anorexia, nausea, 
Table 3 Comparison of the changes of white blood cells among the three groups $\left(\times 10^{9} / \mathrm{L}, \bar{x} \pm s\right)$

\begin{tabular}{lccccccc}
\hline Groups & Before & $5^{\text {th }}$ & $8^{\text {th }}$ & $11^{\text {th }}$ & $14^{\text {th }}$ & $21^{\text {st }}$ \\
\hline Control $(n=55)$ & $7.43 \pm 2.53$ & $6.65 \pm 2.43$ & $5.85 \pm 2.17^{*}$ & $5.50 \pm 2.34$ & $5.37 \pm 2.12^{\text {th }}$ & $5.98 \pm 2.18$ & $6.26 \pm 2.32$ \\
Medication $(n=76)$ & $7.16 \pm 3.10$ & $6.23 \pm 2.56$ & $6.48 \pm 2.88$ & $5.62 \pm 2.21$ & $6.60 \pm 3.24^{\star}$ & $6.13 \pm 2.17$ & $4.47 \pm 2.44$ \\
TEAS $(\mathrm{n}=47)$ & $7.22 \pm 2.54$ & $6.67 \pm 1.85$ & $7.07 \pm 2.10^{\star}$ & $6.32 \pm 2.01$ & $6.11 \pm 1.51^{*}$ & $6.60 \pm 1.88$ & $6.75 \pm 2.30$ \\
F & 0.158 & 0.612 & 5.257 & 1.614 & 7.323 & 0.667 \\
P & 0.854 & 0.544 & 0.023 & 0.203 & 0.026 & 0.515 & 0.715 \\
\hline
\end{tabular}

*, $\mathrm{P}<0.05 ;{ }^{\triangle}, \mathrm{P}<0.05$. White blood cell counts over time for the control, medication, and TEAS groups. The white blood cell in the TEAS group was significantly higher than the control group on day 8 and day $14(P<0.05)$.

Table 4 Comparison of the changes of platelet among the three groups $\left(\times 10^{9} / \mathrm{L}, \bar{x} \pm s\right)$

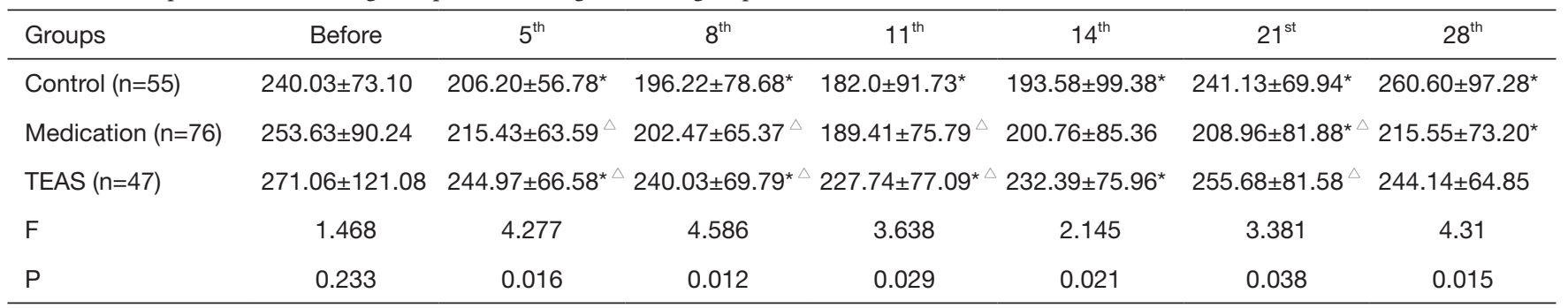

*, $\mathrm{P}<0.05 ;{ }^{\triangle}, \mathrm{P}<0.05$. Platelet counts over time for the control, medication, and TEAS groups. The platelet count in the TEAS group was significantly higher than control group on day 5 , day 8 and day $11(P<0.05)$.

Table 5 Comparison of the changes of red blood cells among the three groups $\left(\times 10^{12} / \mathrm{L}, \bar{x} \pm s\right)$

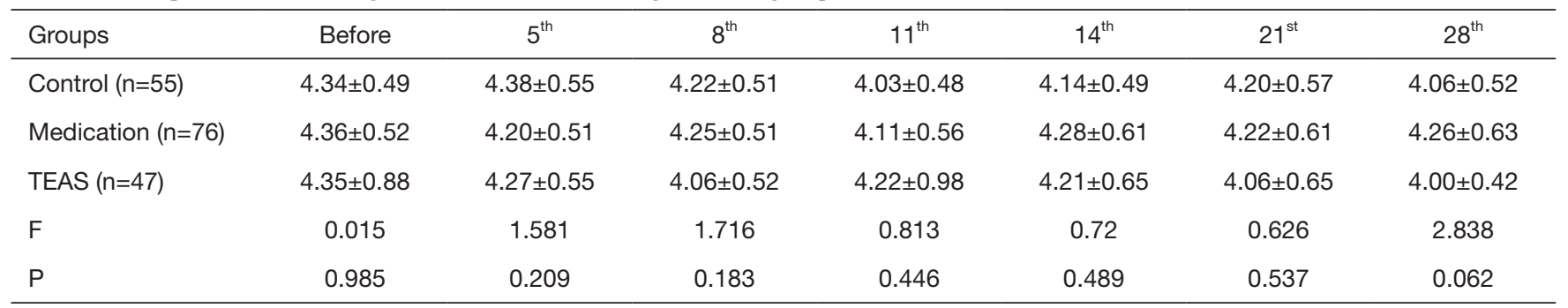

Red blood cell counts over time for the control, medication, and TEAS groups. There were no significant differences in the number of red blood cells between groups on any of the studied time points $(P>0.05)$.

Table 6 Comparison of the changes of hemoglobin among the three groups $(\mathrm{g} / \mathrm{L}, \bar{x} \pm s)$

\begin{tabular}{lccccccc}
\hline Groups & Before & $5^{\text {th }}$ & $8^{\text {th }}$ & $11^{\text {th }}$ & $14^{\text {th }}$ & $21^{\text {st }}$ \\
\hline Control $(\mathrm{n}=55)$ & $129.64 \pm 14.55$ & $128.22 \pm 15.38$ & $124.68 \pm 16.36$ & $120.89 \pm 15.47$ & $122.46 \pm 12.33^{*}$ & $123.13 \pm 12.40$ & $121.20 \pm 14.15$ \\
Medication $(\mathrm{n}=76)$ & $130.71 \pm 14.97$ & $128.27 \pm 15.04$ & $125.97 \pm 12.31$ & $126.28 \pm 14.93$ & $132.13 \pm 34.76^{*}$ & $125.21 \pm 13.78$ & $128.41 \pm 26.65$ \\
TEAS $(\mathrm{n}=47)$ & $125.11 \pm 14.80$ & $125.23 \pm 12.48$ & $122.58 \pm 14.35$ & $121.68 \pm 14.23$ & $122.94 \pm 15.89$ & $124.45 \pm 15.83$ & $120.65 \pm 14.00$ \\
F & 0.218 & 0.62 & 0.663 & 1.858 & 2.397 & 0.273 \\
P & 0.116 & 0.539 & 0.517 & 0.16 & 0.045 & 0.762 & 0.124 \\
\hline
\end{tabular}

${ }^{*}, \mathrm{P}<0.05$. Hemoglobin concentration over time for the control, medication, and TEAS groups. Hemoglobin concentration was significantly higher in the medication groups than in the control group on day $14(\mathrm{P}<0.05)$. 


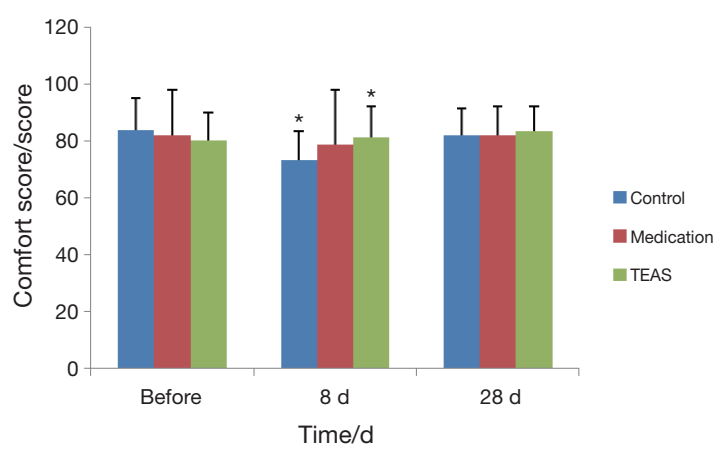

Figure 2 Patient comfort score over time for the control, medication, and TEAS groups. The comfort score in the TEAS group was significantly higher than control group on day $8(\mathrm{P}<0.05)$.

vomiting, and thinning. These symptoms belong to the TCM asthenia category, which includes specific symptoms such as spleen and kidney weakness and deficiency of Qi and blood. Clinical studies have shown that the pathology and pathogenesis after chemotherapy evolve towards the development of spleen deficiency, kidney deficiency, and Qi and blood deficiency (18). In order to control chemotherapy-induced adverse events, this study intended to recuperate spleen and stomach, tonify kidney, and replenish Qi and blood.

TEAS significantly increased white blood cell counts. Dazhui acupoint is located in the seventh cervical spinous process, regulating Qi and blood, and improving white blood cell counts together with acupoint Hegu by $80-90 \%$ (19). Stimulating Zusanli (ST36) induces corticotropin-releasing factors, which enhance the function of the pituitary, adrenal cortex, and sympathetic-adrenal systems and the secretion of vasoactive substances, ultimately adjusting the pressure in the marrow cavity, regulating bone marrow blood flow, and promoting the formation of white blood cells (20). In this study, TEAS increased white blood cell counts. Medication with prophylactic agents also increased white blood cell counts. The white blood cell count was decreased from the beginning of chemotherapy and reached its lowest level on day 14. From day 21 onwards, the white blood cell count began to rise, but the number of white blood cells was higher in the TEAS group than in the control group, indicating that acupuncture point electrical stimulation can increase the white blood cell count.

TEAS significantly increased platelet counts. Electrical stimulation can adjust the platelet count. Shutian et al. stimulated Dazhui, Zusanli, and Sanyinjiao to treat primary thrombocytopenic purpura, and showed that symptoms such as dizziness, fatigue, insomnia, headache, backache, hemorrhage, and ecchymosis were improved significantly. The platelet count also increased significantly to normal level (21). TEAS at Sanyinjiao can nourish Yin and blood. Geshu (located at the seventh thoracic spinous process, 1.5 inches from the median line o) is the rally point of eight points of the blood. Stimulating this acupoint nourishes blood and Qi (22). Studies have shown that acupuncture at Hegu increases platelet count. Stimulating Hegu and Dazhui reduces leukocyte count significantly (23). In this study, TEAS increased platelet counts compared to medication and control groups. Acupoints included Dazhui, Zusanli, Sanyinjiao, and Hegu (LI4). The platelet count was decreased from the beginning of chemotherapy and reached its lowest level on day 11 . On day 14, the platelet count began to rise, but the number of platelets was higher in the TEAS group than in the control groups, indicating that TEAS can play a role in the prevention of thrombocytopenia after chemotherapy.

The effect of TEAS on red blood cell and hemoglobin counts was not obvious. Acupuncture at Geshu significantly increases the number of peripheral white blood cells and enhances bone marrow hematopoietic function (24). Another study has shown that TEAS at Geshu improves the anemia status of anemic rabbits compared to control (25). However, in this study, the effect of TEAS on red blood cells and hemoglobin was not obvious. To observe a significant effect, it may be necessary to increase the sample size or follow up for a longer time, such as more than 120 days. The red blood cell count was decreased from the beginning of chemotherapy and reached its lowest level on day 11 . The number of red blood cells began to increase on day 21 , but was still low until day 28. The same trend was observed for hemoglobin concentration. However, hemoglobin concentration was higher in the medication group than in the control, indicating that the TCM medicine, Diyu Shengbai Pian may be helpful in improving hemoglobin levels in lung cancer patients undergoing chemotherapy.

TEAS can significantly improve the degree of comfort in lung cancer patients receiving chemotherapy. TEAS can improve microcirculation, promote the absorption of inflammatory substances, and improve cell oxygenation and metabolism. It can also promote the recovery of intestinal peristalsis, reduce abdominal distension, and promote defecation. Stimulation of Zusanli and Sanyinjiao can treat vomiting, abdominal distension, constipation, stomachache, lower limb arthralgia, and insomnia $(26,27)$. TEAS can promote blood circulation to remove blood stasis, so as to 
adjust the viscera function and enhance the body's immune function. The meridian theory of TCM shows that stimulation of the relevant acupuncture points of the meridian system can balance Yin and Yang, support healthy and resist evil, so as to achieve the efficacies of promoting blood circulation to remove blood stasis and eliminate inflammation. In this study, TEAS at acupoints Dazhui (28), Geshu, Zusanli, Sanyinjiao, and Hegu (LI4) reduced the side effects of chemotherapy and improved patient comfort. The process of electrical stimulation of the acupuncture points may have favored the communication between nurses and patients, thus narrowing the distance between the two parties.

\section{Conclusions}

Combined electrical stimulation of five acupoints resulted in an increase of the number of white blood cells and platelets and in a reduction of other chemotherapy side effects such as patient discomfort. To confirm the effects of TEAS, a larger, multicenter study is needed. In short, percutaneous electrical stimulation of acupoints could prevent chemotherapy-induced bone marrow suppression in lung cancer patients, thus ensuring a smooth continuation of chemotherapy treatment.

\section{Acknowledgements}

We acknowledge the efforts of the staff of Medical Record Room in the record retrieval.

Funding: The study was supported by a grant from the National Nature and Science Foundation of China (Grant No. Nur81202750). The funding agency has no involvement with the design of the study and collection, analysis, interpretation of data and writing the manuscript.

\section{Footnote}

Conflicts of Interest: The authors have no conflicts of interest to declare.

Ethical Statement: This study was approved by the ethics committee of the Shanghai Pulmonary Hospital, China. Individual consent was not required as this was a secondary data study using a pseudo-anonymised database extract.

\section{References}

1. Chen W, Zheng R, Zhang S, et al. Report of incidence and mortality in China cancer registries, 2009. Chin J Cancer
Res 2013;25:10-21.

2. Jemal A, Bray F, Center MM, et al. Global cancer statistics. CA Cancer J Clin 2011;61:69-90.

3. Andriani F, Perego P, Carenini N, et al. Increased Sensitivity to Cisplatin in Non-Small Cell Lung Cancer Cell Lines after FHIT Gene Transfer. Neoplasia 2006;8:9-17.

4. Gronberg BH, Sundstrom S, Kaasa S, et al. Influence of comorbidity on survival, toxicity and health-related quality of life in patients with advanced non-small-cell lung cancer receiving platinum-doublet chemotherapy. Eur J Cancer 2010;46:2225-34.

5. Smith KM, Jeske CS, Young B, et al. Prevalence and characteristics of adverse drug reactions in neurosurgical intensive care patients. Neurosurgery 2006;58:426-33.

6. Ganti AK, Loberiza FR Jr, Kessinger A. Factors affecting bone marrow toxicity following administration of carboplatin and paclitaxel in patients with non-small cell lung cancer. Anticancer Res 2010;30:1365-9.

7. Liou SY, Stephens JM, Carpiuc KT, et al. Economic burden of haematological adverse effects in cancer patients: a systematic review. Clin Drug lnvestig 2007;27:381-96.

8. Wang XH, Zhu J, Zhao D. Pharmacoeconomic research on adverse reactions of chemotherapy for lung cancer. J Shanghai Jiaotong Univ Med Sci 2012;32:657-9.

9. Berghmans T, Lafitte JJ, Lecomte J, et al. Second-line paclitaxel in non-small cell lung cancer initially treated with cisplatin: a study by the European Lung Cancer Working Party. Br J Cancer 2007;96:1644-9.

10. Zhang QF, Li LX, Lin GH, et al. Effect of direct moxibustion at Sihua points on cytokine of chemotherapy patients with lung cancer (Chinese). Zhongguo Zhen Jiu 2013;33:207-10.

11. Zheng HG, Piao BK. The discussion on etiology of TCM of chemotherapy and radiotherapy (Chinese). Chinese J Bas Med Tradit Chin Med 2007;13:751-2.

12. Zhang Y, Tang XD, Liu YQ. The effect of acupoint injection in the treatment of patients with gynecological tumors to reduce the effect of chemotherapy on bone marrow suppression (Chinese). Modern J Integr Tradit Chin West Med 2013;20:3344-5.

13. Ni F, Lin JY, Zhou CQ, et al. Study on mechanisms of acupoint-injection therapy (Chinese). Chin Acupuncture \& Moxibustion 2003;23:609-11.

14. Dou JQ, Zhang H, Gao WB, et al. clinical study on Acupoint-injecting Method for Marrow-Inhibition Caused by chemical Medications (Chinese). Joural of Acupunture and Tuina Science 2006;4:84-6.

15. Gao WB, Xu B, Du M, et al. Clinical observation 
on acupoint-needling injection of dexamethasone for prevention of arrest of bone marrow induced by chemoautotrophic drugs (Chinese). Chin Clin Oncology 2005;10:281-3.

16. Kolcaba KY. Holistic comfort: operationalizing the construct as a nurse sensitive outcome. ANS Adv Nurs Sci 1992;15:1-10.

17. Zhu LX, Gao FL, Liang XK, et al. Study of comfort status and related factors of post-thoracic surgery patients within 72 hours. Chin J Nurs 2007;42:225-8.

18. Zhang YK. The clinical research on acupoint sticking therapy of Chinese herbal medicines for prevention and treatment of toxic and side-effects arrest of bone marrow induced by chemotherapeutic drugs (Chinese). Fujian J Tradit Chin Med 2003;14:16-8.

19. Tao HF. Acupuncture, moxibustion of Dazhui, Pishu, Zusanli for treating 81 cases of white blood cells decreased (Chinese). J Clin Acupuncture Moxibustion 2003;19:12-3.

20. Wang XY, Wang J, Wu FD. On the location and special application of Zusanli point in Neijing (Chinese). J Clin Acupuncture Moxibustion 2011;27:51-2.

21. Jia SX, Gao CE, Meng GD. Qi Yong Sheng Bai San treating 239 tumor patients with leuco(cyto)penia induced by chemotherapy and radiology (Chinese). Chin J Integr Tradit West Med 1997;17:215-7.

Cite this article as: Hou L, Gu F, Gao G, Zhou C. Transcutaneous electrical acupoint stimulation (TEAS) ameliorates chemotherapy-induced bone marrow suppression in lung cancer patients. J Thorac Dis 2017;9(3):809-817. doi: $10.21037 /$ jtd.2017.03.12
22. Shen DZ, Xiong FL, Cui J. The clinical application of diaphragm acupoints (Chinese). Shanghai J Acupuncture Moxibustion 2004;23:46-8.

23. Li CQ. Professor Li Shi-zhen's clinical experiences on compatibility application of Hegu (LI 4) (Chinese). Zhongguo Zhen Jiu 2010;30:152-4.

24. Cui J, Shen DZ, Xiong FL. Regulating effect of point Geshu acupuncture and moxibustion on leukocyte and marrow hematopoiesis in a rat model of leukopenia (Chinese). Shanghai J Acupuncture and Moxibustion 2005;24:41-3.

25. Shi XM. Science of Acupuncture and Moxibustion. Beijing: China Traditional Chinese Medicine Press 2002;149-50.

26. He XM, Xie XL, Huang W. The effect of Liqi Kuanchang decoction combined with acupoint injection on gastrointestinal tumor patients' comfort after chemotherapy (Chinese). J Qilu Nursing 2015;21:93-5.

27. Bian XM, Yang YW, Zhu YN. The effect of bed exercise combined with acupoint massage on the comfort of patients after prostatectomy (Chinese). Nursing Rehab J 2010;9:420-3.

28. Li J, Zhang L, Jin LC. The effect of acupoint massage in improving comfort degree of patients with coronary intervention operation (Chinese). Shanghai Nursing 2010;10:54-6. 